\title{
BOUNDARY VALUE PROBLEM FOR A SINGULARLY PERTURBED SYSTEM OF LINEAR DIFFERENTIAL EQUATIONS WITH IMPULSES
}

\author{
by D. D. BAINOV, ${ }^{*}$ M. A. HEKIMOVA* and V. M. VELIOV $\dagger$
}

(Received 20th June 1986)

\section{Introduction}

In connection with the analysis of mathematical models of real processes undergoing short time perturbations, in the last years the interest in the differential equations with impulses remarkably increased. Going back to the papers of Mil'man and Myshkis [4, 5] the investigations of this subject are now extended to different directions concerning applications in physics, biology, electronics, automatic control etc.

On the other hand, the description of many dynamic systems calls for very high-order mathematical models due to the presence of small physical parameters like time constants, masses, inductances, capacitances and similar "parasitic" parameters. Often such systems exhibit "slow" and "fast" phenomena simultaneously and are stiff for computations. The suppression of the small parameters in this case leads to a lower order system and therefore the perturbations presented by these parameters are called singular. Starting from the fundamental result of Tichonov [6] a great number of papers have been published developing the singular perturbation approach alleviating both dimensionality and stiffness difficulties. This approach is especially useful in control theory of singularly perturbed systems (see the overview of Kokotovic [3]), where many new problems concerning such systems arise, as well.

The present paper is devoted to a boundary value problem for a linear singularly perturbed system (containing "slow" variables and stable and unstable "fast" variables) with impulses. An asymptotic procedure for solving this problem is constructed (Section 3) and justified (Section 4) by means of a specific modification of the method of boundary-layer functions proposed in Vasileva and Butuzov [7]. Similar results are obtained in Hehimova and Bainov [2] for periodic singularly perturbed systems with impulses.

The boundary value problem considered in the paper arises not only in case of modelling processes which undergo impulse actions, but also in solving optimal control problems for singularly perturbed systems with a criterion depending on the state at some fixed moments of time. The reason is that in this case the adjoint system is also singularly perturbed, but containing impulses at these moments. A control problem of this type is considered in Section 5, where a solution technique is proposed, based on the asymptotic approximations developed in the previous sections. 


\section{Problem statement}

Let $t_{1}<t_{2}<\cdots<t_{p}$ be given points in the open interval $(0, T)$. Consider the following system of linear differential equations with impulses:

$$
\begin{gathered}
\varepsilon \frac{d x}{d t}=A_{1}(t) x+B_{1}(t) y+f_{1}(t), \quad t \neq t_{i} \\
\frac{d y}{d t}=A_{2}(t) x+B_{2}(t) y+f_{2}(t), \quad t \neq t_{i} \\
\Delta x\left(t_{i}\right)=P^{(i)} x\left(t_{i}\right)+a_{i}, \quad \Delta y\left(t_{i}\right)=S^{(i)} y\left(t_{i}\right)+b_{i},
\end{gathered}
$$

where $t \in[0, T],(x, y) \in \mathbb{R}^{m} \times \mathbb{R}^{n}, A_{j}(t), B_{j}(t), f_{j}(t)(j=1,2)$ and $P^{(i)}, S^{(i)}, a_{i}, b_{i}(i=1, \ldots, p)$ are matrices (or vectors) with appropriate dimensions, $\varepsilon$ is a small positive parameter presenting the singular perturbation. We use the notation $\Delta x\left(t_{i}\right)=x\left(t_{i}+0\right)-x\left(t_{i}-0\right)$, $\Delta y\left(t_{i}\right)=y\left(t_{i}+0\right)-y\left(t_{i}-0\right)$, supposing for convenience that all the functions considered are continuous from the left, namely $x\left(t_{i}-0\right)=x\left(t_{i}\right), y\left(t_{i}-0\right)=y\left(t_{i}\right)$.

Let the boundary conditions for the system (1) be given by

$$
x_{1}(0)=x_{1}^{0}, \quad x_{2}(T)=x_{2}^{0}, \quad y(0)=y^{0},
$$

where $x$ is presented as $x=\left(x_{1}, x_{2}\right), x_{1} \in \mathbb{R}^{k}, x_{2} \in \mathbb{R}^{m-k}, \theta \leqq k \leqq m$.

Setting $\varepsilon=0$ in (1) we obtain the so-called reduced system

$$
\begin{gathered}
0=A_{1}(t) \bar{x}+B_{1}(t) \bar{y}+f_{1}(t), \quad t \neq t_{i} \\
\frac{d \bar{y}}{d t}=A_{2}(t) \bar{x}+B_{2}(t) \bar{y}+f_{2}(t), \quad t \neq t_{i} \\
\Delta \bar{y}\left(t_{i}\right)=S^{(i)} \bar{y}\left(t_{i}\right)+b_{i}
\end{gathered}
$$

with the initial condition

$$
\bar{y}(0)=y^{0} .
$$

Further we use the following notation.

1. Given a vector $\mathbf{z}=\left(z_{1}, \ldots, z_{q}\right)$ and $(q \times r)$-matrix $A=\left\{a_{i j}\right\}$ we denote

$$
|z|=\max _{1 \leqq i \leqq q}\left|z_{i}\right|, \quad|A|=\max _{1 \leqq i \leqq q} \sum_{j=1}^{r}\left|a_{i j}\right| .
$$

2. By $\tilde{C}_{q}[0, T]$ we denote the space of all piecewise continuous functions $w:[0, T] \rightarrow \mathbb{R}^{q}$, eventually discontinuous at the points $t_{1}, \ldots, t_{p}$, but continuous from the left. This space will be normed by $\|w\|=\sup \{|w(t)|: t \in[0, T]\}$. 
3. By $\bar{C}_{q}^{(1)}[0, T]$ we denote the set of all functions $w(\cdot) \in \bar{C}_{q}[0, T]$ with a continuous derivative $\dot{w}(\cdot)$ existing on each interval $\left(t_{i}, t_{t+1}\right], i=0,1, \ldots, p+1$ (where $t_{0}=0, t_{p+1}=T$ ), and such that the right derivative $\dot{w}\left(t_{i}+0\right)$ also exists for $i=0, \ldots, p+1$.

Let the following conditions be fulfilled.

A1. The matrices $A_{j}(\cdot), B_{j}(\cdot), j=1,2$, are $\mathcal{N}+1$ times continuously differentiable;

A2. The matrix $A_{1}(t)$ has the following block-diagonal form:

$$
A_{1}(t)=\left(\begin{array}{cc}
A_{11}(t) & 0 \\
0 & A_{22}(t)
\end{array}\right)
$$

where $A_{11}(t)$ is a $(k \times k)$-matrix with strictly negative real parts of its eigenvalues $\left(\operatorname{Re} \lambda\left(A_{11}(t)\right)<0\right)$, while for the $(m-k) \times(m-k)$-matrix $A_{22}(t)$ the real parts of the eigenvalues are positive $\left(\operatorname{Re} \lambda\left(A_{22}(t)\right)>0\right)$;

A3. Presenting the matrices $P^{(i)}, i=1, \ldots, p$ in the block-diagonal form

$$
P^{(i)}=\left(\begin{array}{ll}
P_{11}^{(i)} & P_{12}^{(i)} \\
P_{21}^{(i)} & P_{22}^{(i)}
\end{array}\right)
$$

according to the block-diagonal form of $A_{1}(t)$, we assume that the matrices $E+S^{(i)}$, $E+P_{11}^{(i)}$ and $E+P_{22}^{(i)}, i=1, \ldots, p$, are non-singular ( $E$ is the identity matrix);

A4. The functions $f_{j}(\cdot), j=1,2$, belong to $\bar{C}[0, T]$.

Under the conditions A1-A4 we prove the existence of a solution of the boundary value problem (1), (2). We construct also an asymptotic expansion of this solution with respect to the small parameter $\varepsilon$, including terms in a "stretched time scale" modelling the fast movement of the trajectory near the boundary and near the moments $t_{i}$ of impulse actions. The expansion of the solution of (1), (2) $z(t, \varepsilon)=(x(t, \varepsilon), y(t, \varepsilon))$ takes the form

$$
z(t, \varepsilon)=\bar{z}(t, \varepsilon)+\pi^{(i i)} z\left(\tau_{i}, \varepsilon\right)+Q^{(i)} z\left(\sigma_{i}, \varepsilon\right), \quad t_{i}<t \leqq t_{i+1}
$$

where $z(t, \varepsilon)$ and the boundary-layer functions $\pi^{(i)} z(\cdot, \varepsilon)$ and $Q^{(i)} z(\cdot, \varepsilon)$ are presented as series:

$$
\begin{gathered}
\bar{z}(t, \varepsilon)=\sum_{k=0}^{\infty} \varepsilon^{k} z_{k}(t), \quad t \in[0, T], \\
\pi^{(i)} z\left(\tau_{i}, \varepsilon\right)=\sum_{k=0}^{\infty} \varepsilon^{k} \pi_{k}^{(i)} z\left(\tau_{i}\right) \quad(i=0, \ldots, p), \\
Q^{(i)} z\left(\sigma_{i}, \varepsilon\right)=\sum_{k=0}^{\infty} \varepsilon^{k} Q_{k}^{(i)} z\left(\sigma_{i}\right) \quad(i=0, \ldots, p),
\end{gathered}
$$

Here $\tau_{i}=\left(t-t_{i}\right) / \varepsilon$ and $\sigma_{i}=\left(t-t_{i+1}\right) / \varepsilon, t \in\left(t_{i}, t_{i+1}\right)$ are the stretched time scales. The 
additional condition

$$
\pi_{k}^{(i)} z(+\infty)=0, \quad Q_{k}^{(i)} z(-\infty)=0, \quad i=0, \ldots, p, \quad k=0,1, \ldots
$$

for the boundary-layer functions yields their local influence on the solution approximation. An algorithm constructing the above expansions is described in Section 3. The convergence of the series obtained is proved in Section 4, where an estimation of the difference in the uniform metric between the solution and its $\mathcal{N}$ th approximation is also found.

\section{Formal asymptotic expansion of the solution}

First, we shall find an appropriate formal asymptotic expansion of the solution $z(t, \varepsilon)=(x(t, \varepsilon), y(t, \varepsilon))$ of the boundary value problem (1), (2) in the form given in (6)-(9).

The reduced problem (3), (4) can be presented as

$$
\begin{gathered}
\bar{x}(t)=\phi(t, \bar{y}), \quad t \neq t_{t} \\
\dot{y}(t)=\left[B_{2}(t)-A_{2}(t) A_{1}^{-1}(t) B_{1}(t)\right] \bar{y}(t)+F(t), \quad t \neq t_{i} \\
\Delta \bar{y}\left(t_{i}\right)=S^{(i)} \bar{y}\left(t_{i}\right)+b_{i},
\end{gathered}
$$

where

$$
\begin{gathered}
\phi(t, y)=-A_{1}^{-1}(t) B_{1}(t) y-A_{1}^{-1}(t) f_{1}(t), \\
F(t)=f_{2}(t)-A_{2}(t) A_{1}^{-1}(t) f_{1}(t) .
\end{gathered}
$$

System (12) with initial condition (4) has a unique solution given by

$$
\bar{y}(t)=V(t, 0) y^{0}+\int_{0}^{t} V(t, s) F(s) d s+\sum_{0<t_{v}<t} V\left(t, t_{v}+0\right) b_{v},
$$

where $V(t, s)$ is the fundamental matrix solution of $(15)$, normalized at $t=s$ :

$$
\begin{gathered}
\dot{\zeta}=\left(B_{2}(t)-A_{2}(t) A_{1}^{-1}(t) B_{1}(t)\right) \zeta, \quad t \neq t_{i} \\
\Delta \zeta\left(t_{i}\right)=S^{(i)} \zeta\left(t_{i}\right) .
\end{gathered}
$$

Substituting (6) in system (1) we obtain

$$
\begin{aligned}
\varepsilon \frac{d \bar{x}}{d t}+ & \frac{d \pi^{(i)} x}{d \tau_{i}}+\frac{d Q^{(i)} x}{d \sigma_{i}} \\
= & A_{1}(t) \bar{x}(t, \varepsilon)+A_{1}\left(t_{i}+\varepsilon \tau_{i}\right) \pi^{(i)} x\left(\tau_{i}, \varepsilon\right)+A_{1}\left(t_{i+1}+\varepsilon \sigma_{i}\right) Q^{(i)} x\left(\sigma_{i}, \varepsilon\right) \\
& +B_{1}(t) \bar{y}(t, \varepsilon)+B_{1}\left(t_{i}+\varepsilon \tau_{i}\right) \pi^{(i)} y\left(\tau_{i}, \varepsilon\right)+B_{1}\left(t_{i+1}+\varepsilon \tau_{i}\right) Q^{(i)} y\left(\sigma_{i}, \varepsilon\right)+f_{1}(t), \\
& t_{i}<t \leqq t_{i+1}, \quad i=0,1, \ldots, p,
\end{aligned}
$$




$$
\begin{aligned}
\varepsilon \frac{d \bar{y}}{d t}+ & \frac{d \pi^{(i)} y}{d \tau_{i}}+\frac{d Q^{(i)} y}{d \sigma_{i}} \\
= & \varepsilon A_{2}(t) \bar{x}(t, \varepsilon)+\varepsilon A_{2}\left(t_{i}+\varepsilon \tau_{i}\right) \pi^{(i)} x\left(\tau_{i}, \varepsilon\right)+\varepsilon A_{2}\left(t_{i+1}+\varepsilon \sigma_{i}\right) Q^{(i)} x\left(\sigma_{i}, \varepsilon\right) \\
& +\varepsilon B_{2}(t) \bar{y}(t, \varepsilon)+\varepsilon B_{2}\left(t_{i}+\varepsilon \tau_{i}\right) \pi^{(i)} y\left(\tau_{i}, \varepsilon\right)+\varepsilon B_{2}\left(t_{i+1}+\varepsilon \sigma_{i}\right) Q^{(i)} y\left(\sigma_{i}, \varepsilon\right)+\varepsilon f_{2}(t), \\
& t_{i}<t \leqq t_{i+1}, \quad i=0,1, \ldots, p
\end{aligned}
$$

and from the impulse action equalities in (1)

$$
\begin{aligned}
& \bar{x}\left(t_{i}+0, \varepsilon\right)+\pi^{(i)} x(0, \varepsilon)+Q^{(i)} x\left(\frac{t_{i}-t_{i+1}}{\varepsilon}, \varepsilon\right) \\
& =\left(P^{(i)}+E\right)\left[\bar{x}\left(t_{i}, \varepsilon\right)+\pi^{(i-1)} x\left(\frac{t_{i}-t_{i-1}}{\varepsilon}, \varepsilon\right)+Q^{(i-1)} x(0, \varepsilon)\right]+a_{i}, \\
& \bar{y}\left(t_{i}+0, \varepsilon\right)+\pi^{(i)} y(0, \varepsilon)+Q^{(i)} y\left(\frac{t_{i}-t_{i+1}}{\varepsilon}, \varepsilon\right) \\
& =\left(S^{(i)}+E\right)\left[\bar{y}\left(t_{i}, \varepsilon\right)+\pi^{(i-1)} y\left(\frac{t_{i}-t_{i-1}}{\varepsilon}, \varepsilon\right)+Q^{(i)} y(0, \varepsilon)\right]+b_{i} .
\end{aligned}
$$

In the above equations (16) and (17) we replace $\bar{z}, \pi^{(i)} z$ and $Q^{(i)} z$ by the expressions (7), (8) and (9) and present the matrices $A_{j}\left(t_{i}+\varepsilon \tau_{i}\right), A_{j}\left(t_{i+1}+\varepsilon \sigma_{i}\right), B_{j}\left(t_{i}+\varepsilon \tau_{i}\right)$ and $B_{j}\left(t_{i+1}+\varepsilon \sigma_{i}\right)$ $(j=1,2)$ as power series with respect to $\varepsilon$. Comparing the coefficients of each power of $\varepsilon$ (these depending on $t, \tau_{i}$ and $\sigma_{i}$, separately) we obtain a system of equations with respect to the coefficients in (7), (8) and (9). Substituting (6) in (2) we get the boundary conditions

$$
\begin{aligned}
& x_{1}^{0}=\sum_{k=0}^{\infty} \varepsilon^{k} \bar{x}_{k, 1}(0)+\sum_{k=0}^{\infty} \varepsilon^{k} \pi_{k, 1}^{(0)} x(0)+\sum_{k=0}^{\infty} \varepsilon^{k} Q_{k, 1}^{(0)} x\left(-\frac{t_{1}}{\varepsilon}\right) \\
& x_{2}^{0}=\sum_{k=0}^{\infty} \varepsilon^{k} \bar{x}_{k, 2}(T)+\sum_{k=0}^{\infty} \varepsilon^{k} \pi_{k, 2}^{(p)} x\left(\frac{T-t_{p}}{\varepsilon}\right)+\sum_{k=0}^{\infty} \varepsilon^{k} Q_{k, 2}^{(p)}(0) x(0), \\
& y^{0}=\sum_{k=1}^{\infty} \varepsilon^{k} \bar{y}_{k}(0)+\sum_{k=0}^{\infty} \varepsilon^{k} \pi_{k}^{(0)} y(0)+\sum_{k=0}^{\infty} \varepsilon^{k} Q_{k}^{(0)} y\left(-\frac{t_{1}}{\varepsilon}\right)
\end{aligned}
$$

where the additional indices 1 and 2 appear due to the separation of the fast state to a stable and an unstable part, namely $x=\left(x_{1}, x_{2}\right) \in \mathbb{R}^{k} \times \mathbb{R}^{m-k}$. 
For the first term $\left(\bar{x}_{0}(t), \bar{y}_{0}(t)\right)$ we obtain the system

$$
\begin{array}{cc}
0=A_{1}(t) \bar{x}_{0}+B_{1}(t) \bar{y}_{0}+f_{1}(t), & t \neq t_{i} \\
\dot{y}_{0}=A_{2}(t) \bar{x}_{0}+B_{2}(t) \bar{y}_{0}+f_{2}(t), \quad t \neq t_{i} \\
\Delta \bar{y}_{0}\left(t_{i}\right)=S^{(i)} \bar{y}_{0}\left(t_{i}\right)+b_{i} .
\end{array}
$$

The boundary-layer functions $\pi_{0}^{(i)} z\left(\tau_{i}\right)$ and $Q_{0}^{(i)} z\left(\sigma_{i}\right)$ satisfy

$$
\begin{gathered}
\frac{d \pi_{0}^{(i)} x}{d \tau_{i}}=A_{1}\left(t_{i}\right) \pi_{0}^{(i)} x\left(\tau_{i}\right)+B_{1}(t) \pi_{0}^{(i)} y\left(\tau_{i}\right) ; \quad \frac{d \pi_{0}^{(i)} y}{d \tau_{i}}=0 \\
\frac{d Q_{0}^{(i)} x}{d \sigma_{i}}=A_{1}\left(t_{i+1}\right) Q_{0}^{(i)} x\left(\sigma_{i}\right)+B_{1}\left(t_{i+1}\right) Q_{0}^{(i)} y\left(\sigma_{i}\right) ; \quad \frac{d Q_{0}^{(i)} y}{d \sigma_{i}}=0 .
\end{gathered}
$$

From (20) and (21), taking into account (10) we have

$$
\pi_{0}^{(i)} y\left(\tau_{i}\right) \equiv 0, Q_{0}^{(i)} y\left(\sigma_{i}\right) \equiv 0
$$

Using the block-diagonal form of the matrix $A_{1}$ (condition A2) we replace (20) and (21) by

$$
\begin{aligned}
& \frac{d \pi_{0,1}^{(i)} x}{d \tau_{i}}=A_{11}\left(t_{i}\right) \pi_{0,1}^{(i)} x\left(\tau_{i}\right), \\
& \frac{d \pi_{0,2}^{(i)} x}{d \tau_{i}}=A_{22}\left(t_{i}\right) \pi_{0,2}^{(i)} x\left(\tau_{i}\right), \\
& \frac{d Q_{0,1}^{(i)} x}{d \sigma_{i}}=A_{11}\left(t_{i+1}\right) Q_{0,1}^{(i)} x\left(\sigma_{i}\right), \\
& \frac{d Q_{0,2}^{(i)} x}{d \sigma_{i}}=A_{22}\left(t_{i+1}\right) Q_{0,2}^{(i)} x\left(\sigma_{i}\right) .
\end{aligned}
$$

From (18) and (22) we find the initial condition

$$
\bar{y}_{\mathrm{o}}(0)=y^{0}
$$

for the system (19). Thus the problem (19), (27) is exactly the reduced problem (3), (4) and $\bar{x}_{0}(t)=\phi\left(t, \bar{y}_{0}(t)\right), \bar{y}_{0}(t)=\bar{y}(t)$.

From (10) and (24), (25), using the spectrum condition in A2 we conclude that $\pi_{0,2}^{(i)} x(\cdot) \equiv 0, Q_{0,1}^{(i)} x(\cdot) \equiv 0, i=0, \ldots, p$. 
The equalities (18) and (17) give us

$$
\begin{gathered}
\pi_{0,1}^{(0)} x(0)=x_{1}^{0}-\bar{x}_{0,1}(0), \quad Q_{0,2}^{(p)} x(0)=x_{2}^{0}-\bar{x}_{0,2}(T), \\
Q_{0,2}^{(i)} x(0)=\left(P_{22}^{(i+1)}+E\right)^{-1}\left[\bar{x}_{0,2}\left(t_{i+1}+0\right)-\left(P_{21}^{(i+1)}+E\right) \bar{x}_{0,1}\left(t_{i+1}\right)-a_{i+1,2}\right] \\
-\bar{x}_{0,2}\left(t_{i+1}\right), \quad i=0, \ldots, p-1, \\
\pi_{0,1}^{(i)} x(0)=\left(P_{11}^{(i)}+E\right) \bar{x}_{0,1}\left(t_{i}\right)+\left(P_{12}^{(i)}+E\right)\left[\bar{x}_{0.2}\left(t_{i}\right)+Q_{0,2}^{(i-1)} x(0)\right] \\
+a_{i, 1}-\bar{x}_{0,1}\left(t_{i}+0\right), \quad i=1, \ldots, p .
\end{gathered}
$$

For the solutions of the systems (23) and (26) with initial conditions (30) and (29) we have

$$
\begin{array}{cc}
\pi_{0,1}^{(i)} x\left(\tau_{i}\right)=\pi_{0,1}^{(i)} x(0) e^{A_{11}\left(t_{i}\right) \tau_{i}}, & \tau_{i} \geqq 0, \\
Q_{0,2}^{(i)} x\left(\sigma_{i}\right)=Q_{0,2}^{(i)} x(0) e^{A_{22\left(t_{i}+1\right) \sigma_{i}},} & \sigma_{i} \leqq 0 .
\end{array}
$$

The condition A2 implies the existence of constants $C$ and $\kappa>0$ such that

$$
\left\|\pi_{0}^{(i)} x\left(\tau_{i}\right)\right\| \leqq C e^{-\kappa \tau_{i}}, \tau_{i} \geqq 0 ; \quad\left\|Q_{0}^{(i)} x\left(\sigma_{i}\right)\right\| \leqq C e^{\kappa \sigma_{i}}, \sigma_{i} \leqq 0 ; \quad i=1, \ldots, p .
$$

In a similar way we can find the coefficients of $\varepsilon^{k}(k \geqq 1)$ in (6), (7) and (8). As a result we obtain the systems

$$
\begin{gathered}
\dot{x}_{k-1}(t)=A_{1}(t) \bar{x}_{k}(t)+B_{1}(t) \bar{y}_{k}(t), \quad t \neq t_{i} \\
\dot{y}_{k}(t)=A_{2}(t) \bar{x}_{k}(t)+B_{2}(t) \bar{y}_{k}(t), \quad t \neq t_{i} \\
\Delta \bar{y}_{k}\left(t_{i}\right)=S^{(i)} \bar{y}_{k}\left(t_{i}\right)+\left(S^{(i)}+E\right) Q_{k}^{(i-1)} y(0)-\pi_{k}^{(i)} y(0) \\
\frac{d \pi_{k}^{(i)} x}{d \tau_{i}}=A_{1}\left(t_{i}\right) \pi_{k}^{(i)} x\left(\tau_{i}\right)+B_{1}\left(t_{i}\right) \pi_{k}^{(i)} y\left(\tau_{i}\right)+T_{k}^{(i)}\left(\tau_{i}\right) \\
\frac{d \pi_{k}^{(i)} y}{d \tau_{i}}=R_{k}^{(i)}\left(\tau_{i}\right) \\
\frac{d Q_{k}^{(i)} x}{d \sigma_{i}}=A_{1}\left(t_{i+1}\right) Q_{k}^{(i)} x\left(\sigma_{i}\right)+B_{1}\left(t_{i+1}\right) Q_{k}^{(i)} y\left(\sigma_{i}\right)+G_{k}^{(i)}\left(\sigma_{i}\right) \\
\frac{d Q_{k}^{(i)} y}{d \sigma_{i}}=
\end{gathered}
$$


where

$$
\begin{aligned}
& T_{k}^{(i)}\left(\tau_{i}\right)=\sum_{s=1}^{k} \frac{\tau_{i}^{s}}{s !}\left[\frac{d^{s} A_{1}\left(t_{i}\right)}{d t^{s}} \pi_{k-s}^{(i)} x\left(\tau_{i}\right)+\frac{d^{s} B_{1}\left(t_{i}\right)}{d t^{s}} \pi_{k-s}^{(i)} y\left(\tau_{i}\right)\right] \\
& R_{k}^{(i)}\left(\tau_{i}\right)=\sum_{s=0}^{k-1} \frac{\tau_{i}^{s}}{s !}\left[\frac{d^{s} A_{2}\left(t_{i}\right)}{d t^{s}} \pi_{k-s-1}^{(i)} x\left(\tau_{i}\right)+\frac{d^{s} B_{2}\left(t_{i}\right)}{d t^{s}} \pi_{k-s-1}^{(i)} y\left(\tau_{i}\right)\right] \\
& G_{k}^{(i)}\left(\sigma_{i}\right)=\sum_{s=1}^{k} \frac{\sigma_{i}^{s}}{s !}\left[\frac{d^{s} A_{1}\left(t_{i+1}\right)}{d t^{s}} Q_{k-s}^{(i)} x\left(\sigma_{i}\right)+\frac{d^{s} B_{1}\left(t_{i+1}\right)}{d t^{s}} Q_{k-s}^{(i)} y\left(\sigma_{i}\right)\right] \\
& H_{k}^{(i)}\left(\sigma_{i}\right)=\sum_{s=0}^{k-1} \frac{\sigma_{i}^{s}}{d t^{s}}\left[\frac{d^{s} A_{2}\left(t_{i+1}\right)}{d t^{s}} Q_{k-s-1}^{(i)} x\left(\sigma_{i}\right)+\frac{d^{s} B_{2}\left(t_{i+1}\right) d t^{s}}{d t^{s}} Q_{k-s-1}^{(i)} y\left(\sigma_{i}\right)\right]
\end{aligned}
$$

The initial conditions for $\pi_{k}^{(i)} y\left(\tau_{i}\right), Q_{k}^{(i)} y\left(\sigma_{i}\right), i=1, \ldots, p, k=1, \ldots, \mathcal{N}$ are given according to (10) as follows:

$$
\pi_{k}^{(i)} y(0)=-\int_{0}^{\infty} R_{k}^{(i)}(s) d s, \quad Q_{k}^{(i)} y(0)=-\int_{0}^{-\infty} H_{k}^{(i)}(s) d s .
$$

From the second equations in (35) and (36) and from (38) we get

$$
\pi_{k}^{(i)} y\left(\tau_{i}\right)=-\int_{\tau_{i}}^{\infty} R_{k}^{(i)}(s) d s, \quad Q_{k}^{(i)} y\left(\sigma_{i}\right)=-\int_{\sigma_{i}}^{-\infty} H_{k}^{(i)}(s) d s .
$$

Taking into account (38) we rewrite system (34) in the form

$$
\begin{gathered}
\bar{x}_{k}(t)=\phi_{k}\left(t, \bar{y}_{k}(t)\right), \quad t \neq t_{i} \\
\hat{y}_{k}(t)=\left[B_{2}(t)-A_{2}(t) A_{1}^{-1}(t) B_{1}(t)\right] \bar{y}_{k}+F_{k}(t), \quad t \neq t_{i} \\
\Delta y_{k}\left(t_{i}\right)=S^{(i)} \bar{y}_{k}\left(t_{i}\right)+\gamma_{k}^{(i)}
\end{gathered}
$$

with the initial condition

$$
\bar{y}_{k}(0)=-\pi_{k}^{(0)} y(0)
$$

obtained from (18), where

$$
\begin{gathered}
\phi_{k}\left(t, \bar{y}_{k}(t)\right)=-A_{1}^{-1}(t) B_{1}(t) \bar{y}_{k}(t)+A_{1}^{-1}(t) \dot{x}_{k-1}(t), \quad t \neq t_{i}, \\
F_{k}(t)=A_{2}(t) A_{1}^{-1}(t) \bar{x}_{k-1}(t), \\
\gamma_{k}^{(i)}=-\left(S^{(i)}+E\right) \int_{0}^{-\infty} H_{k}^{(i-1)}(s) d s+\int_{0}^{+\infty} R_{k}^{(i)}(s) d s .
\end{gathered}
$$


The solution $\left(\bar{x}_{k}(t), \bar{y}_{k}(t)\right)$ of $(40),(41)$ is given by

$$
\begin{gathered}
\bar{x}_{k}(t)=\phi_{k}\left(t, \bar{y}_{k}(t)\right) \\
\bar{y}_{k}(t)=-V(t, 0) \pi_{k}^{(0)} y(0)+\int_{0}^{t} V(t, s) F_{k}(s) d s+\sum_{0<t_{v}<t} V\left(t, t_{v}+0\right) \gamma_{k}^{(v)} .
\end{gathered}
$$

Using (10) we obtain from (18) the initial conditions for $\pi_{k}^{(i)} x\left(\tau_{i}\right)$ and $Q_{k}^{(i)} x\left(\sigma_{i}\right)$ :

$$
\begin{aligned}
\pi_{k, 1}^{(0)} x(0)= & -\bar{x}_{k, 1}(0) \\
\pi_{k, 1}^{(i)} x(0)= & \left(P_{11}^{(i)}+E\right) \bar{x}_{k, 1}\left(t_{i}\right)+\left(P_{12}^{(i)}+E\right)\left[\bar{x}_{k, 2}\left(t_{i}\right)+Q_{k, 2}^{(i-1)} x(0)\right] \\
& -\bar{x}_{k, 1}\left(t_{i}+0\right), \quad i=\overline{1, p} \\
\pi_{k, 2}^{(i)} x(0)= & -\int_{0}^{+\infty} e^{-A_{22}\left(t_{i+1}\right) s}\left[B_{1}\left(t_{i}\right) \pi_{k}^{(i)} y(s)+T_{k}^{(i)}(s)\right]_{1} d s, \quad i=\overline{0, p} \\
Q_{k, 1}^{(i)} x(0)= & -\int_{0}^{-\infty} e^{-A_{11}\left(t_{i+1}\right) s}\left[B_{1}\left(t_{i+1}\right) Q_{k}^{(i)} y(s)+G_{k}^{(i)}(s)\right]_{1} d s, \quad i=\overline{0, p}, \\
Q_{k, 2}^{(i)} x(0)= & \left(P_{22}^{(i+1)}+E\right)^{-1}\left[\bar{x}_{k, 2}\left(t_{i+1}+0\right)-\left(P_{21}^{(i+1)}+E\right) \bar{x}_{k, 1}\left(t_{i+1}\right)\right] \\
& -\bar{x}_{k, 2}\left(t_{i+1}\right), \quad i=\overline{0, p-1} \\
Q_{k, 2}^{(p)}(x(0)= & -\bar{x}_{k, 2}(T) .
\end{aligned}
$$

Using condition A2 we obtain from the system (36)

$$
\begin{aligned}
& \frac{d Q_{k, 1}^{(i)} x}{d \sigma_{i}}=A_{11}\left(t_{i+1}\right) Q_{k, 1}^{(i)} x\left(\sigma_{i}\right)+\left[B_{1}\left(t_{i+1}\right) Q_{k}^{(i)} y\left(\sigma_{i}\right)+G_{k}^{(i)}\left(\sigma_{i}\right)\right]_{1} \\
& \frac{d Q_{k, 2}^{(i)}}{d \sigma_{i}}=A_{22}\left(t_{i+1}\right) Q_{k, 2}^{(i)} x\left(\sigma_{i}\right)+\left[B_{1}\left(t_{i+1}\right) Q_{k}^{(i)} y\left(\sigma_{i}\right)+G_{k}^{(i)}\left(\sigma_{i}\right)\right]_{2}
\end{aligned}
$$

with (44) as an initial condition. Using the Cauchy formula we get

$$
\begin{aligned}
Q_{k, 1}^{(i)} x\left(\sigma_{i}\right)= & -\int_{\sigma_{i}}^{-\infty} e^{A_{11}\left(t_{i+1}\right)\left(\sigma_{i}-s\right)}\left[B_{1}\left(t_{i+1}\right) Q_{k}^{(i)} y(s)+G_{k}^{(i)}(s)\right]_{1} d s, \\
Q_{k, 2}^{(i)} x\left(\sigma_{i}\right)= & Q_{k, 2}^{(i)} x(0) e^{A_{22}\left(t_{i+1}\right) \sigma_{i}}+\int_{0}^{\sigma_{i}} e^{A_{22\left(t_{i+1}\right)\left(\sigma_{i}-s\right)}}\left[B_{1}\left(t_{i+1}\right) Q_{k}^{(i)} y(s)\right. \\
& \left.+G_{k}^{(i)}(s)\right]_{2} d s, \quad i=0, \ldots, p, \quad \sigma_{i} \leqq 0 .
\end{aligned}
$$


Finally, solving the problem (35), (43) with $Q_{k, 2}^{(i-1)}(0)$ obtained from (45) we get for $\tau_{i} \geqq 0$

$$
\begin{aligned}
& \pi_{k, 1}^{(i)} x\left(\tau_{i}\right)=\pi_{k, 1}^{(i)} x(0) e^{A_{11}\left(t_{i}\right) \tau_{i}}+\int_{0}^{\tau_{i}} e^{A_{11}\left(t_{i j}\right)\left(\tau_{i}-s\right)}\left[B_{1}\left(t_{i}\right) \pi_{k}^{(i)} y(s)+T_{k}^{(i)}(s)\right]_{1} d s, \\
& \pi_{k, 2}^{(i)} x\left(\tau_{i}\right)=-\int_{\tau_{i}}^{\infty} e^{A_{22}\left(t_{i}\right)\left(\tau_{i}-s\right)}\left[B_{1}\left(t_{i}\right) \pi_{k}^{(i)} y(s)+T_{k}^{(i)}(s)\right]_{2} d s
\end{aligned}
$$

Thus, the coefficients in the expansions (7), (8) and (9) are completely determined. Moreover, using (33) we prove by induction that there exist constants $C$ and $\kappa>0$ such that the boundary-layer functions $\pi_{k}^{(i)} z\left(\tau_{i}\right)$ and $Q_{k}^{(i)} z\left(\sigma_{i}\right)(k=0, \ldots, \mathcal{N}, i=1, \ldots, p)$ satisfy the estimations

$$
\left\|\pi_{k}^{(i)} z\left(\tau_{i}\right)\right\| \leqq C e^{-\kappa \tau_{i}}, \tau_{i} \geqq 0 ; \quad\left\|Q_{k}^{(i)} z\left(\sigma_{i}\right)\right\| \leqq C e^{\kappa \sigma_{i}}, \sigma_{i} \leqq 0
$$

(here and further we use the letters $C$ and $\kappa$ to indicate constants, which however, may be specific in different relations).

The estimations (47) imply the convergence of all the integrals on infinite intervals encountered above. Thus, the formal expansions (7), (8) and (9) are correctly defined. The question of the convergence of these series will be investigated in the next section.

\section{Convergence of the asymptotic expansion and existence of a solution}

The main purpose of this section is to prove the convergence of the power series (6) (described in (7), (8) and (9)) to a function $z(t, \varepsilon)$ being a solution of the problem (1), (2). Moreover, we estimate the difference between the exact solution and the $\mathcal{N}$ th approximation

$$
\left.Z_{\mathscr{N}}(t, \varepsilon)=\sum_{k=0}^{\mathscr{N}} \varepsilon^{k}\left[\bar{z}_{k}(t)+\pi_{k}^{(i)} z\left(\tau_{i}\right)+Q_{k}^{(i)} z \sigma_{i}\right)\right], \quad t_{i}<t \leqq t_{i+1}
$$

in the uniform metric.

First we shall consider the following "fast" system with impulses

$$
\begin{gathered}
\varepsilon \dot{r}=A_{1}(t) r+\psi(t), \quad t \neq t_{i} \\
\Delta r\left(t_{i}\right)=P^{(i)} r\left(t_{i}\right)+c_{i}
\end{gathered}
$$

together with the boundary conditions

$$
r_{1}(0, \varepsilon)=r_{1}^{0}, \quad r_{2}(T, \varepsilon)=r_{2}^{0}
$$

where as above $r=\left(r_{1}, r_{2}\right) \in \mathbb{R}^{k} \times \mathbb{R}^{m-k}$. This problem turns out to have a solution and this solution will be estimated under the following conditions. 
B1. The matrix $A_{1}(t)$ is continuous and satisfies the condition $\mathrm{A} 2$ in $[0, T]$ :

B2. The matrices $P_{11}^{(i)}+E$ and $P_{22}^{(i)}+E, i=1, \ldots, p$, are nonsingular;

B3. The vector function $\psi(t)$ belongs to $\tilde{C}_{m}[0, T]$.

Thus the system (48) consists of the following two systems, coupled by the impulse relations only:

$$
\begin{gathered}
\varepsilon \dot{r}_{1}=A_{11} r_{1}+\psi_{1}(t), \quad t \neq t_{i} \\
\Delta r_{1}\left(t_{i}\right)=P_{11}^{(i)} r_{1}\left(t_{i}\right)+P_{12}^{(i)} r_{2}\left(t_{i}\right)+c_{i, 1}
\end{gathered}
$$

and

$$
\begin{gathered}
\varepsilon \dot{r}_{2}=A_{22} r_{2}+\psi_{2}(t), \quad t \neq t_{i} \\
\Delta r_{2}\left(t_{i}\right)=P_{22}^{(i)} r_{2}\left(t_{i}\right)+P_{21}^{(i)} r_{1}\left(t_{i}\right)+c_{i, 2} .
\end{gathered}
$$

Denote by $\Psi_{k}(t, s, \varepsilon)\left(\Psi_{k}(s, s, \varepsilon)=E\right), k=1,2$ the fundamental matrix solution of the system

$$
\varepsilon \dot{\zeta}_{k}=A_{k k} \zeta_{k}, \quad t \in[0, T]
$$

without impulses. It is well known (see [1]) that under the condition B1 the following inequalities hold

$$
\begin{aligned}
\left|\Psi_{1}(t, s, \varepsilon)\right| \leqq K_{0} e^{-\kappa(t-s / \varepsilon)}, & 0 \leqq s \leqq t \leqq T, \\
\left|\Psi_{2}(t, s, \varepsilon)\right| \leqq K_{0} e^{\kappa(t-s / \varepsilon)}, & 0 \leqq t \leqq s \leqq T,
\end{aligned}
$$

where $K_{0}$ and $\kappa>0$ are appropriate constants. Denote

$$
\begin{aligned}
& U_{1}(t, s, \varepsilon)= \begin{cases}\Psi_{1}(t, s, \varepsilon), & t_{i}<s \leqq t \leqq t_{i+1}, \\
\Psi_{1}\left(t, t_{i}, \varepsilon\right)\left(E+P_{11}^{(i)}\right) \Psi_{1}\left(t_{i}, s, \varepsilon\right), & t_{i-1}<s \leqq t_{i} \leqq t_{i+1}, \\
\Psi_{1}\left(t, t_{i}, \varepsilon\right)\left[\prod_{j=i}^{k+1}\left(E+P_{11}^{(j)}\right) \Psi_{1}\left(t_{j}, t_{j-1}, \varepsilon\right)\right]\left(E+P_{11}^{(k)}\right) & \\
\quad \times \Psi_{1}\left(t_{k}, s, \varepsilon\right), \quad t_{k-1}<s \leqq t_{k}<t_{i}<t \leqq t_{i+1},\end{cases} \\
& U_{2}(t, s, \varepsilon)=\left\{\begin{array}{c}
\Psi_{2}(t, s, \varepsilon), t_{i}<t \leqq s \leqq t_{i+1}, \\
\Psi_{2}\left(t, t_{i}, \varepsilon\right)\left(E+P_{22}^{(i)}\right)^{-1} \Psi_{2}\left(t_{i}, s, \varepsilon\right), t_{i-1}<t \leqq t_{i}<s \leqq t_{i+1} \\
\Psi_{2}\left(t, t_{i}, \varepsilon\right)\left[\prod_{j=i}^{k-1}\left(E+P_{22}^{(j)}\right)^{-1} \Psi_{2}\left(t_{j}, t_{j+1}, \varepsilon\right)\right]\left(E+P_{22}^{(k)}\right)^{-1} \\
\quad \times \Psi_{2}\left(t_{k}, s, \varepsilon\right), t_{i-1}<t \leqq t_{i}<t_{k}<s \leqq t_{i+1} .
\end{array}\right.
\end{aligned}
$$


By direct calculations we see that $U_{k}(t, s, \varepsilon), k=1,2$, is the fundamental matrix solution of the corresponding homogeneous system with impulses

$$
\begin{gathered}
\varepsilon \dot{\eta}_{k}=A_{k k}(t) \eta_{k}, \quad t \neq t_{i} \\
\Delta \eta_{k}\left(t_{i}\right)=P_{k k}^{(i)} \eta_{k}\left(t_{i}\right) .
\end{gathered}
$$

The estimations (53) and the presentations (54) and (55) yield the existence of constants $K, \varepsilon_{0}>0$ and $\kappa>0$ such that for $\varepsilon \in\left(0, \varepsilon_{0}\right]$

$$
\begin{array}{ll}
\left|U_{1}(t, s, \varepsilon)\right| \leqq K e^{-\kappa(t-s / \varepsilon)}, & 0 \leqq s \leqq t \leqq T, \\
\left|U_{2}(t, s, \varepsilon)\right| \leqq K e^{\kappa(t-s / \varepsilon)}, & 0 \leqq t \leqq s \leqq T .
\end{array}
$$

Consider the system $(50)$ under the additional assumption that $P_{12}^{(i)}=0(i=1, \ldots, p)$, and with the initial condition

$$
r_{1}(0, \varepsilon)=r_{1}^{0}
$$

Lemma 1. Let conditions B1-B3 hold. Then there exist $\varepsilon_{0}>0$ and a constant $K_{1}$ such that for every $\varepsilon \in\left(0, \varepsilon_{0}\right]$ and $t \in[0, T]$ the unique solution $\tilde{r}_{1}(t, \varepsilon)$ of the system $(50)$ with the initial condition (58) and with $P_{12}^{(i)}=0(i=1, \ldots, p)$ satisfies the inequality

$$
\left\|\tilde{r}_{1}(t, \varepsilon)\right\| \leqq K_{1} \max \left\{\left\|\psi_{1}\right\|, \max _{i}\left|c_{i, 1}\right|,\left|r_{1}^{0}\right|\right\}
$$

Proof. The solution of (50), (58) with $P_{12}^{(i)}=0$ is given by

$$
\tilde{r}_{1}(t, \varepsilon)=U_{1}(t, 0, \varepsilon) r_{1}^{0}+\frac{1}{\varepsilon} \int_{0}^{t} U_{1}(t, s, \varepsilon) \psi_{1}(s) d s+\sum_{0<t_{v}<t} U_{1}\left(t, t_{v}+0, \varepsilon\right) c_{v, 1}
$$

Then inequality (59) follows from the first inequality in (57) and (60).

Lemma 2. Let conditions B1-B3 hold. Then there exist $\varepsilon_{0}>0$ and a constant $K_{2}$ such that for every $\varepsilon \in\left(0, \varepsilon_{0}\right]$ and $t \in[0, T]$ the solution $\tilde{r}_{2}(t, \varepsilon)$ of the system (51) with $P_{21}^{(i)}=0$ $(i=1, \ldots, p)$ resulting from the condition

$$
\tilde{r}_{2}(T, \varepsilon)=r_{2}^{0}
$$

satisfies the inequality

$$
\left\|\tilde{r}_{2}(t, \varepsilon)\right\| \leqq K_{2} \max \left\{\left\|\psi_{2}\right\|, \max _{i}\left|c_{i, 2}\right|,\left|r_{2}^{0}\right|\right\}
$$


Proof. We directly establish that the function

$$
\tilde{r}_{2}(t, \varepsilon)=U_{2}(t, T, \varepsilon) r_{2}^{0}+\frac{1}{\varepsilon} \int_{T}^{t} U_{2}(t, s, \varepsilon) \psi_{2}(s) d s-\sum_{t \leqq t_{v}<T} U_{2}\left(t, t_{v}+0, \varepsilon\right)\left(E+P_{22}^{(v)}\right)^{-1} c_{v, 2}
$$

belongs to $\tilde{C}_{m-k}[0, T]$ and satisfies (51) and (61). Then the estimation (62) follows from the second inequality in (57) and (63).

Lemma 3. Let the conditions $\mathrm{B} 1-\mathrm{B} 3$ hold. Then there exists $\varepsilon_{0}>0$ such that for $\varepsilon \in\left(0, \varepsilon_{0}\right]$ system $(48)$ with boundary conditions $(49)$ has a unique solution $r(\cdot, \varepsilon) \in \bar{C}_{m}[0, T]$. This solution satisfies the inequality

$$
\|r(t, \varepsilon)\| \leqq K_{3} \max \left\{\|\psi\|, \max _{i}\left|c_{i}\right|,\left|r^{0}\right|\right\}
$$

where $K_{3}$ is an appropriate constant.

Proof. Denote by $r_{1}\left(t ; \varepsilon ; r_{2}\left(t_{1}\right), \ldots, r_{2}\left(t_{p}\right)\right)$ the solution of the system $(50)$ with the initial condition (58), where $r_{2}\left(t_{1}\right), \ldots, r_{2}\left(t_{p}\right)$ are considered as parameters. Denote, further, by $r_{2}\left(t ; \varepsilon ; r_{2}\left(t_{1}\right), \ldots, r_{2}\left(t_{p}\right)\right)$ the solution of the system (51) with the condition (61), where $r_{1}\left(t_{i}\right)$ is replaced by $r_{1}\left(t_{i} ; \varepsilon ; r_{2}\left(t_{1}\right), \ldots, r_{2}\left(t_{p}\right)\right), i=1, \ldots, p$. In orders to obtain a solution of (48), (49) it remains to find the parameters $r_{2}\left(t_{1}\right), \ldots, r_{2}\left(t_{p}\right)$ such that the following system of linear algebraic equations is satisfied

$$
r_{2}\left(t_{i} ; \varepsilon ; r_{2}\left(t_{1}\right), \ldots, r_{2}\left(t_{p}\right)\right)=r_{2}\left(t_{i}\right), \quad(i=1, \ldots, p)
$$

Using the estimations (57) we easily conclude that the determinant $\Delta(\varepsilon)$ of this system can be presented as

$$
\Delta(\varepsilon)=E+\Delta_{1}(\varepsilon)
$$

where $\Delta_{1}(\varepsilon)$ tends to zero together with $\varepsilon$. Hence system (65) possesses a unique solution $r_{2}^{0}\left(t_{1}\right), \ldots, r_{2}^{0}\left(t_{p}\right)$ for all sufficiently small $\varepsilon$. Then obviously $r_{1}(t, \varepsilon)=r_{1}\left(t ; \varepsilon ; r_{2}^{0}\left(t_{1}\right), \ldots, r_{2}^{0}\left(t_{p}\right)\right)$ and $r_{2}(t, \varepsilon)=r_{2}\left(t ; \varepsilon ; r_{2}^{0}\left(t_{1}\right), \ldots, r_{2}^{0}\left(t_{p}\right)\right)$ give a solution of problem (48), (49). The estimation (64) follows from (59) and (62). The proof is complete.

Theorem. Let conditions A1-A3 hold. Then there exist constants $M$ and $\varepsilon_{0}>0$ such that for every $\varepsilon \in\left(0, \varepsilon_{0}\right]$ the boundary value problem (1), (2) has a unique solution $z(t, \varepsilon)$ and this solution satisfies the inequality

$$
\left\|z(t, \varepsilon)-Z_{\mathcal{N}}(t, \varepsilon)\right\| \leqq M \varepsilon^{\mathcal{N}+1}, \quad t \in[0, T]
$$

Proof. Introducing the new variables

$$
u=x-X_{\mathcal{N}}(t, \varepsilon), \quad v=y-Y_{\mathcal{N}}(t, \varepsilon)
$$


we transform (1) to the system

$$
\begin{array}{cc}
\varepsilon \dot{u}=A_{1}(t) u+B_{1}(t) v+g_{1}(t, \varepsilon), & t \neq t_{i} \\
\dot{v}=\left(B_{2}(t)-A_{2}(t) A_{1}^{-1}(t) B_{1}(t)\right) v+D(t)\left[A_{1}(t) u+B_{1}(t) v\right]+g_{2}(t, \varepsilon), & t \neq t_{i} \\
\Delta u\left(t_{1}\right) P^{(i)} u\left(t_{i}\right)+\alpha_{i}(\varepsilon) & \\
\Delta v\left(t_{i}\right)=S^{(i)} v\left(t_{i}\right)+\beta_{i}(\varepsilon) &
\end{array}
$$

where

$$
\begin{gathered}
D(t)=A_{2}(t) A_{1}^{-1}(t), \\
g_{1}(t, \varepsilon)=A_{1}(t) X_{\mathcal{N}}(t, \varepsilon)+B_{1}(t) Y_{\mathcal{N}}(t, \varepsilon)-\varepsilon \frac{d X_{\mathcal{N}}}{d t}+f_{1}(t), \\
g_{2}(t, \varepsilon)=A_{2}(t) X_{\mathcal{N}}(t, \varepsilon)+B_{2}(t) Y_{\mathcal{N}}(t, \varepsilon)-\frac{d Y_{\mathcal{N}}}{d t}+f_{2}(t), \\
\alpha_{i}(\varepsilon)=\left(P^{(i)}+E\right)\left[\prod_{s=0}^{\mathscr{N}} \varepsilon^{s} \pi_{s}^{(i-1)} x\left(\frac{t_{i}-t_{i-1}}{\varepsilon}\right)-\sum_{s=0}^{\mathscr{N}} \varepsilon^{s} Q_{s}^{(i)} x\left(\frac{t_{i}-t_{i+1}}{\varepsilon}\right)\right], \\
\beta_{i}(\varepsilon)=\left(S^{(i)}+E\right)\left[\sum_{s=0}^{\mathcal{N}} \varepsilon^{s} \pi_{s}^{(i-1)} y\left(\frac{t_{i}-t_{i-1}}{\varepsilon}\right)-\sum_{s=0}^{\mathscr{N}} \varepsilon^{s} Q_{s}^{(i)} y\left(\frac{t_{i}-t_{i+1}}{\varepsilon}\right)\right] .
\end{gathered}
$$

From (2) and (67) we obtain the following boundary conditions for system (68)

$$
u_{1}(0, \varepsilon)=p_{1}(\varepsilon), \quad u_{2}(T, \varepsilon)=p_{2}(\varepsilon), \quad v(0, \varepsilon)=q(\varepsilon)
$$

where

$$
\begin{gathered}
p_{1}(\varepsilon)=-\sum_{k=1}^{\mathscr{N}} \varepsilon^{k} Q_{k, 1}^{(0)} x\left(-\frac{t_{1}}{\varepsilon}\right) ; \quad p_{2}(\varepsilon)=-\sum_{k=1}^{\mathscr{N}} \varepsilon^{k} \pi_{k, 2}^{(p)} x\left(\frac{T-t_{p}}{\varepsilon}\right), \\
q(\varepsilon)=-\sum_{k=1}^{\mathcal{N}} \varepsilon^{k} Q_{k}^{(0)} y(0) .
\end{gathered}
$$

From the relations (19) $-(21),(27)-(30),(34)-(38),(41),(43),(44)$ and (47) we conclude that the estimations

$$
\begin{gathered}
|D(t)| \leqq N_{0}, \quad\left|g_{1}(t, \varepsilon)\right| \leqq N_{1} \varepsilon^{\mathcal{r}+1}, \quad t \in[0, T], \\
\mid g_{2}(t, \varepsilon) \leqq N_{2} \varepsilon^{\mathcal{V}}\left[e^{-\kappa\left(t-t_{i} / \varepsilon\right)}+e^{\kappa\left(t-t_{i+1} / \varepsilon\right)}\right], \quad t_{i}<t \leqq t_{i+1}, \quad i=1, \ldots, p,
\end{gathered}
$$




$$
\begin{gathered}
\left|\alpha_{i}(\varepsilon)\right| \leqq N_{3} \varepsilon^{\mathcal{V}+1}, \quad \mid \beta_{i}(\varepsilon) \leqq N_{4} \varepsilon^{\mathcal{V}+1}, \quad i=1, \ldots, p, \\
\left|p_{k}(\varepsilon)\right| \leqq N_{5} \varepsilon^{\mathcal{N}+1}(k=1,2), \quad \mid q(\varepsilon) \leqq N_{6} \varepsilon^{\mathcal{N}+1}
\end{gathered}
$$

hold for all sufficiently small $\varepsilon>0\left(N_{j}, j=0, \ldots, 6\right.$ are appropriate constants).

Given a constant $\rho>0$, define the set

$$
T_{\rho}=\left\{w: w \in \tilde{C}_{n}[0, T],\|w\| \leqq \rho\right\} .
$$

Lemma 3 implies that the system

$$
\begin{gathered}
\varepsilon \dot{h}=A_{1}(t) h+B_{1}(t) w+g_{1}(t, \varepsilon), \quad t \neq t_{i} \\
\Delta h\left(t_{i}\right)=P^{(i)} h\left(t_{i}\right)+\alpha_{i}(\varepsilon)
\end{gathered}
$$

with boundary conditions

$$
h_{1}(0, \varepsilon)=p_{1}(\varepsilon), \quad h_{2}(T, \varepsilon)=p_{2}(\varepsilon)
$$

has a unique solution $h(t, w, \varepsilon) \in \tilde{C}_{m}[0, T]$, when $w \in T_{\rho}$. Then relations (64) and (72) yield the existence of constants $\varepsilon_{0}>0, L_{0}, L_{1}$ such that

$$
\begin{gathered}
\|h(t, w, \varepsilon)\| \leqq L_{0}\|w\|+L_{1} \varepsilon^{\mathcal{r}+1}, \quad w \in T_{\rho}, \\
\left\|h\left(t, w_{1}, \varepsilon\right)-h\left(t, w_{2}, \varepsilon\right)\right\| \leqq L_{0}\left\|w_{1}-w_{2}\right\|, \quad w_{1}, w_{2} \in T_{\rho}
\end{gathered}
$$

for $\varepsilon \in\left(0, \varepsilon_{0}\right]$.

For $w \in T_{\rho}$ we denote by $\phi_{\varepsilon} w$ the solution of the following system

$$
\begin{gathered}
\dot{\tilde{v}}=\left(B_{2}(t)-A_{2}(t) A_{1}^{-1}(t) B_{1}(t)\right) \tilde{v}+D(t)\left[A_{1}(t) h(t, w, \varepsilon)\right. \\
\left.+B_{1}(t) w(t)\right]+g_{2}(t, \varepsilon), \quad t \neq t_{i} \\
\Delta \tilde{v}\left(t_{i}\right)=S^{(i)} \tilde{v}\left(t_{i}\right)+\beta_{i}(\varepsilon)
\end{gathered}
$$

with the initial condition

$$
\tilde{v}(0, \varepsilon)=q(\varepsilon)
$$

Then

$$
\phi_{\varepsilon} w=\int_{0}^{t} V(t, s) D(s)\left[A_{1}(s) h(s)+B_{1}(s) w(s)\right] d s+G_{2}(t, \varepsilon),
$$

where $V(t, s)$ is the fundamental matrix solution of system (15) and

$$
G_{2}(t, \varepsilon)=V(t, 0) q(\varepsilon)+\int_{0}^{t} V(t, s) g_{2}(s, \varepsilon) d s+\sum_{0<t_{v}<t} V\left(t, t_{v}+0\right) \beta_{v}(\varepsilon) .
$$


From (79), taking into account that $h(t, w, \varepsilon)$ solves (73), we get

$$
\phi_{\varepsilon} w=\varepsilon \int_{0}^{t} V(t, s) D(s) \dot{h}(s) d s-\int_{0}^{t} V(t, s) D(s) g_{1}(s, \varepsilon) d s+G_{2}(t, \varepsilon),
$$

where $\dot{h}\left(t_{i}\right)$ means $\dot{h}\left(t_{i}-0\right)$. The first term in the right-hand side of (80) can be written as

$$
\begin{aligned}
\varepsilon \int_{0}^{t} V(t, s) D(s) \dot{h}(s) d s \\
=\varepsilon \int_{0}^{t_{1}} V(t, s) D(s) \dot{h}(s) d s \\
\quad+\sum_{i=1}^{k-1} \varepsilon \int_{t_{i}}^{t_{i+1}} V(t, s) D(s) \dot{h}(s) d s+\varepsilon \int_{i_{k}}^{t} V(t, s) D(s) \dot{h}(s) d s,
\end{aligned}
$$

where $0<t_{1}<\cdots<t_{k}<t \leqq T$. Integrating by parts (81) we have

$$
\begin{aligned}
\varepsilon \int_{0}^{t} V(t, s) D(s) \dot{h}(s) d s \\
=\varepsilon D(t) h(t)-\varepsilon V(t, 0) D(0) h(0) \\
\quad+\varepsilon \sum_{i=1}^{k}\left[V\left(t, t_{i}\right) D\left(t_{i}\right)-V\left(t, t_{i}+0\right) D\left(t_{i}\right)\left(E+P^{(i)}\right)\right] h\left(t_{i}\right) \\
\quad-\varepsilon \sum_{i=1}^{k} V\left(t, t_{i}+0\right) D\left(t_{i}\right) \alpha_{i}(\varepsilon)-\varepsilon \int_{0}^{t} \frac{\partial(V(t, s)) \cdot D(s)}{\partial s} h(s) d s .
\end{aligned}
$$

From (82), using the estimations (72) and the boundedness of the matrices $V(t, s)$ and $\partial(V(t, s) D(s)) / \partial s$ for $0 \leqq s \leqq t \leqq T$, we obtain that

$$
\left|\varepsilon \int_{0}^{1} V(t, s) D(s) \dot{h}(s) d s\right| \leqq \varepsilon \mathcal{N}_{7}\|h\|+\mathscr{N}_{8} \varepsilon^{\mathcal{N}+2}
$$

for $0 \leqq t \leqq T, \varepsilon \in\left(0, \varepsilon_{0}\right], \mathscr{N}_{7}, \mathscr{N}_{8}$-appropriate constants.

The second term in (80) and the matrix $G_{2}(t, \varepsilon)$ can be estimated also by means of (72):

$$
\begin{gathered}
\left|\int_{0}^{t} V(t, s) D(s) g_{1}(s, \varepsilon) d s\right| \leqq \mathcal{N}_{9} \varepsilon^{\mathcal{N}+1}, \\
\mid G_{2}(t, \varepsilon) \leqq \mathcal{N}_{10} \varepsilon^{\mathcal{N}+1}
\end{gathered}
$$

for $0 \leqq t \leqq T, \varepsilon \in\left(0, \varepsilon_{0}\right], \mathcal{N}_{9}, \mathcal{N}_{10}$-constants. Using (80), (83), (84) and (85) we obtain

$$
\left\|\phi_{\varepsilon} w\right\| \leqq \varepsilon \mathcal{N}_{7}\|h\|+\mathscr{N}_{11} \varepsilon^{\mathcal{r + 1}}
$$

for $w \in T_{\rho}, \varepsilon \in\left(0, \varepsilon_{0}\right], \mathscr{N}_{11}-$ a constant. 
Similarly we prove the inequality

$$
\left\|\phi_{\varepsilon} w_{1}-\phi_{\varepsilon} w_{2}\right\| \leqq N_{12} \varepsilon\left\|h_{1}-h_{2}\right\|
$$

for $w_{1}, w_{2} \in T_{\rho}, h_{k}=h\left(t, w_{k}, \varepsilon\right)(k=1,2), \varepsilon \in\left(0, \varepsilon_{0}\right], N_{12}$-a constant.

From estimations (75), (76), (86) and (87) we conclude that $\phi_{\varepsilon}$ is a contractive operator in $T_{\rho}$. Denoting by $v(t, \varepsilon)$ the unique fixed point of $\phi_{\varepsilon}$, we obtain that $(u(t, \varepsilon), v(t, \varepsilon)), u(t, \varepsilon)=h(t, v(t, \varepsilon), \varepsilon)$, is the unique solution of $(68),(70)$ for $\varepsilon \in\left(0, \varepsilon_{0}\right]$.

Setting $\rho=C \varepsilon$ with a sufficiently large constant $C$ and using (75) and (86) we get

$$
\|u(t, \varepsilon)\| \leqq M \varepsilon^{v+1}, \quad\|v(t, \varepsilon)\| \leqq M \varepsilon^{r+1} .
$$

The above estimations together with (67) give us estimations (66). The proof is complete.

\section{Applications to control theory}

In this section we apply the asymptotic algorithm presented in Section 2 in solving optimal control problems for singularly perturbed linear systems with a criterion depending on the state of the system at given moments of time. In this case the adjoint system contains impulse actions and together with the original system forms a boundary value problem for a singularly perturbed linear system with impulses.

Consider the following optimal control problem:

$$
\begin{gathered}
\operatorname{minimize} \sum_{i=1}^{p}\left(\left\langle\frac{1}{2} p_{1}^{i} x\left(t_{i}\right)+q_{1}^{i}, x\left(t_{i}\right)\right\rangle+\left\langle\left\langle\frac{1}{2} p_{2}^{i} y\left(t_{i}\right)+q_{2}^{i}, y\left(t_{i}\right)\right\rangle\right)\right. \\
+\frac{1}{2} \int_{0}^{T}(\langle R x(t), x(t)\rangle+\langle Q u(t), u(t)\rangle) d t
\end{gathered}
$$

subject to

$$
\begin{array}{cc}
\dot{x}=A_{1} x+A_{2} y+B_{1} u+f_{1}, & x(0)=x_{0}, \\
\varepsilon \dot{y}=A_{3} x+A_{4} y+B_{2} u+f_{2}, & y(0)=y_{0}, \\
x(T) \in S, &
\end{array}
$$

where $(x, y) \in \mathbb{R}^{m} \times \mathbb{R}^{n}$ is the state, $u \in \mathbb{R}^{r}$ is the control variable, $\varepsilon$ is a small positive parameter providing the singular perturbation, $\langle\cdot, \cdot\rangle$ denotes the scalar product. All the above matrices and vectors have appropriate dimensions and may depend on the time $t$ in a sufficiently smooth way. The moments $t_{1}<\cdots<t_{p}$ are assumed to be from the open interval $(0, T)$.

Let the set $S$ constraining the final state of the "slow" variable $x$ be described by the equations

$$
g_{j}(x)=0, \quad j=1, \ldots, l
$$


where $g_{j}(\cdot)$ are differentiable and let the following be also fulfilled:

C1. The real parts of the eigenvalues of the matrix $A_{4}$ are strictly negative;

C2. The matrices $R$ and $Q$ are positive definite and $Q$ is nonsingular.

We shall briefly describe a numerical procedure for solving the problem (88), (89), which alleviates both dimensionality and stiffness difficulties. The well-known approach of reduction of the original problem (88), (89) to a boundary value problem is used.

Denoting for convenience by $(\xi, \varepsilon \eta) \in \mathbb{R}^{m} \times \mathbb{R}^{n}$ the adjoint variable we can present the adjoint system as

$$
\begin{gathered}
\dot{\xi}=-A_{1}^{*} \xi-A_{3}^{*} \eta+R x+\sum_{i=1}^{p} \delta\left(t_{i}\right)\left(p_{1}^{i} x\left(t_{i}\right)+q_{1}^{i}\right), \xi(T) \in \mathcal{N}(x(T)), \\
\varepsilon \dot{\eta}=-A_{2}^{*} \xi-A_{4}^{*} \eta+\sum_{i=1}^{p} \delta\left(t_{i}\right)\left(p_{2}^{i} y\left(t_{i}\right)+q_{2}^{i}\right), \quad \eta(T)=0,
\end{gathered}
$$

where $\mathscr{N}(x)$ is the subspace of $\mathbb{R}^{m}$ spanned by the vectors $g_{1}^{\prime}(x), \ldots, g_{l}^{\prime}(x)$. Given a vector $(\xi, \varepsilon \eta) \in \mathbb{R}^{m} \times \mathbb{R}^{n}$, the hamiltonian attains its maximum with respect to $u$ at

$$
u=Q^{-1} B_{1}^{*} \xi+Q^{-1} B_{2}^{*} \eta
$$

Thus, the pair of the optimal trajectory and the corresponding adjoint variable satisfies the following system of differential equations and boundary conditions:

$$
\begin{array}{ll}
\dot{x}=A_{1} x+A_{2} y+B_{1} Q^{-1} B_{1}^{*} \xi+B_{1} Q^{-1} B_{l}^{*} \eta+f_{1}, & x(0)=x_{0}, \\
\dot{\xi}=R x-A_{1}^{*} \xi-A_{3}^{*} \eta, & \xi(T) \in \mathcal{N}(x(T)), \\
\varepsilon \dot{y}=A_{3} x+A_{4} y+B_{2} Q^{-1} B_{1}^{*} \xi+B_{2} Q^{-1} B_{2}^{*} \eta+f_{2}, & y(0)=y_{0}, \\
\varepsilon \dot{\eta}=-A_{2}^{*} \xi-A_{4}^{*} \eta, & \eta(T)=0
\end{array}
$$

with impulses at the points

$$
\begin{gathered}
\Delta \xi\left(t_{i}\right)=p_{1}^{(i)} x\left(t_{i}\right)+q_{1}^{(i)} \\
\Delta \eta\left(t_{i}\right)=\frac{1}{\varepsilon}\left[p_{2}^{(i)} y\left(t_{i}\right)+q_{2}^{(i)}\right] .
\end{gathered}
$$

Since the matrix $A_{4}$ is asymptotically stable, one can find a matrix $K$ such that $A_{4} K+K A_{4}^{*}=B_{2} Q^{-1} B_{2}^{*}$. Introducing a new variable $\tilde{y}=y+K \eta$ instead of $y$ we obtain from (90) the following system

$$
\begin{array}{ll}
\dot{x}=A_{1} x+A_{2} \tilde{y}+B_{1} Q^{-1} B_{1}^{*} \xi+\left(B_{1} Q^{-1} B_{2}^{*}-A_{2} K\right) \eta+f_{1}, & x(0)=x_{0}, \\
\dot{\xi}=R x-A_{1}^{*} \xi-A_{3}^{*} \eta, & \xi(T) \in \mathcal{N}(x(T)),
\end{array}
$$




$$
\begin{array}{ll}
\varepsilon \dot{\tilde{y}}=A_{3} x+A_{4} \tilde{y}+B_{2} Q^{-1} B_{1}^{*} \xi+f_{2}, & \tilde{y}(0)=y_{0}+K \eta(0), \\
\varepsilon \dot{\eta}=-A_{2}^{*} \xi-A_{4}^{*} \eta, & \eta(T)=0 .
\end{array}
$$

The stable and the unstable fast variables $\tilde{y}$ and $\eta$ are now separated and thus condition A2 is fulfilled. The impulses (91) take the form

$$
\begin{aligned}
& \Delta \xi\left(t_{i}\right)=p_{1}^{(i)} x\left(t_{i}\right)+q_{1}^{(i)} \\
& \Delta \tilde{y}\left(t_{i}\right)=\frac{1}{\varepsilon}\left[K p_{2}^{(i)} \tilde{y}\left(t_{i}\right)-K p_{2}^{(i)} K \eta\left(t_{i}\right)+K q_{2}^{(i)}\right] \\
& \Delta \eta\left(t_{i}\right)=\frac{1}{\varepsilon}\left[p_{2}^{(i)} \tilde{y}\left(t_{i}\right)-p_{2}^{(i)} K \eta\left(t_{i}\right)+q_{2}^{(i)}\right] .
\end{aligned}
$$

In order to apply the asymptotic expansion developed in Section 2 we have to assume additionally that

C3. $\quad p_{2}^{(i)}=\varepsilon \tilde{p}_{2}^{(i)}, \quad q_{2}^{(i)}=\varepsilon \tilde{q}_{2}^{(i)}, \quad i=1, \ldots, p$.

C4. The matrices $E-p_{2}^{(i)} K$ and $E+K p_{2}^{(i)}$ are nonsingular, $i=1, \ldots, p$.

Remark 1. The assumption $\mathrm{C} 3$ is natural. Often the original system describing the dynamics is given in terms of the state variable $z=y / \varepsilon$ involving large coefficients in the right-hand sides of the corresponding equations. If the state value $z\left(t_{i}\right)$ is penalized similarly as $x\left(t_{i}\right)$ by the criterion, then the normalizing substitution $y=\varepsilon z$ leads to a criterion satisfying the assumption $\mathrm{C} 3$. Moreover, the most practically important case is $p_{2}^{(i)}=0, q_{2}^{(i)}=0$.

Remark 2. The presence of the fact phenomena $y$ in the integral part of the criterion (88) is also admissible, if the corresponding term is multiplied by the factor $\varepsilon_{0}$. The fast subsystem in (92) will be weakly coupled in this case and similar asymptotic techniques can be applied.

In order to solve the boundary value problem (92), (93) we can use some version of the shutting method. Replace the final condition in the second equation in (92) by the initial condition $\xi(0)=\xi_{0}$ and the initial condition in the third equation by $\tilde{y}(0)=y_{0}$ $+K \eta_{0}$. Thus, we obtain a new boundary value problem with impulses which will be denoted by $\mathscr{P}\left(\xi_{0}, \eta_{0}\right)$ (we consider $\xi_{0}$ and $\eta_{0}$ as unknown parameters). Let $(x, y, \xi, \eta)\left(\xi_{0}, \eta_{0} ; t\right)$ be the solution of this problem. Our optimal control problem (88), (89) is now reduced to the following system of $n+m$ equations with respect to $\left(\xi_{0}, \eta_{0}\right) \in \mathbb{R}^{m} \times \mathbb{R}^{n}:$

$$
\begin{gathered}
x\left(\xi_{0}, \eta_{0} ; T\right) \in S, \\
\xi\left(\xi_{0}, \eta_{0} ; T\right) \in \mathcal{N}\left(x\left(\xi_{0}, \eta_{0} ; T\right)\right), \\
\eta\left(\xi_{0}, \eta_{0} ; 0\right)=\eta_{0} .
\end{gathered}
$$


Each step of any iterative procedure for solving the last system requires the solution of the problem $\mathscr{P}\left(\xi_{0}, \eta_{0}\right)$ for some fixed $\xi_{0}, \eta_{0}$. The last problem is exactly in the form of (1), (2) and it is convenient to use the asymptotic expansion described in Section 2. Practically, the first one or two terms of the expansion are enough, but the accuracy can be increased (see estimation (66)) according to the current discrepancy in equations (94).

\section{REFERENCES}

1. L. Flato and N. Levinson, Periodic solutions of singularly perturbed systems, J. Rat. Mech. Anal. 4 (1953), 943-950.

2. M. A. Hekimova and D. D. Bainov, Periodic solutions of singularly perturbed systems of differential equations with impulse effect, $Z A M P 36$ (1985), 520-537.

3. P. V. Кокотоvic, Applications of singular perturbation techniques to control problems, SIAM Rev. 26 (1984), 501-550.

4. V. D. Mil'man and A. D. Myshkis, On the stability of motion in presence of impulses, Siberian Math. J. 1 (1960), 233-237 (in Russian).

5. V. D. Mit'man and A. D. Myshkis, Random impulses in linear dynamic systems, in Asymptotic Methods for Solving Differential Equations (Ed. AN UkSSR, Kiev, 1963), 64-81 (in Russian).

6. A. N. Tironov, Systems of differential equations containing a small parameter multiplying the derivative, Mat. Sb. 31(73) (1952), 575-586.

7. A. B. VASILEVA and V. F. Butuzov, Asymptotic Expansions of Solutions of Singularly Perturbed Systems of Differential Equations (Nauka, Moscow, 1973) (in Russian).

(*)

Department of Mathematics

UNIVERSITY OF PLOVDIV

Plovdiv

Bulgaria
(†)

InSTITUTE OF Mathematics

Bulgarian ACademy of ScIences 1090 Sofia

P.O. Box 373

Bulgaria 